\title{
Inhibition of EZH2 methyltransferase decreases immunoediting of mesothelioma cells by autologous macrophages through a PD-1-dependent mechanism
}

\author{
Malik Hamaidia, ${ }^{1,2}$ Hélène Gazon, ${ }^{1}$ Clotilde Hoyos, ${ }^{1,2}$ Gabriela Brunsting Hoffmann, ${ }^{1,2}$ Renaud Louis, ${ }^{3}$ \\ Bernard Duysinx, ${ }^{3}$ and Luc Willems ${ }^{1,2}$ \\ 'Molecular and Cellular Epigenetics (Groupe Interdisciplinaire de Génoprotéomique Appliquée [CICA]), Liège, Belgium. \\ ${ }^{2}$ Molecular Biology, TERRA, Gembloux, Belgium. ${ }^{3}$ Department of Pneumology, University Hospital of Liège, Liège, Belgium.
}

The roles of macrophages in orchestrating innate immunity through phagocytosis and $T$ lymphocyte activation have been extensively investigated. Much less understood is the unexpected role of macrophages in direct tumor regression. Tumoricidal macrophages can indeed manifest cancer immunoediting activity in the absence of adaptive immunity. We investigated direct macrophage cytotoxicity in malignant pleural mesothelioma, a lethal cancer that develops from mesothelial cells of the pleural cavity after occupational asbestos exposure. In particular, we analyzed the cytotoxic activity of mouse RAW264.7 macrophages upon cell-cell contact with autologous AB1/AB12 mesothelioma cells. We show that macrophages killed mesothelioma cells by oxeiptosis via a mechanism involving enhancer of zeste homolog 2 (EZH2), a histone H3 lysine 27-specific (H3K27-specific) methyltransferase of the polycomb repressive complex 2 (PRC2). A selective inhibitor of EZH2 indeed impaired RAW264.7-directed cytotoxicity and concomitantly stimulated the PD-1 immune checkpoint. In the immunocompetent BALB/c model, RAW264.7 macrophages pretreated with the EZH2 inhibitor failed to control tumor growth of $A B 1$ and AB12 mesothelioma cells. Blockade of PD-1 engagement restored macrophage-dependent antitumor activity. We conclude that macrophages can be directly cytotoxic for mesothelioma cells independent of phagocytosis. Inhibition of the PRC2 EZH2 methyltransferase reduces this activity because of PD-1 overexpression. Combination of $\mathrm{PD}-1$ blockade and $\mathrm{EZH} 2$ inhibition restores macrophage cytotoxicity.

Conflict of interest: The authors have declared that no conflict of interest exists.

Copyright: (c) 2019, American Society for Clinical Investigation.

Submitted: February 27, 2019

Accepted: August 13, 2019

Published: September 19, 2019.

Reference information: /CI Insight. 2019;4(18):e128474.

https://doi.org/10.1172/jici.

insight.128474.

\section{Introduction}

Malignant pleural mesothelioma (MPM) is a highly lethal cancer arising from mesothelial cells of the pleural cavity upon occupational exposure to asbestos (1). MPM pathogenesis is associated with the carcinogenic effect of asbestos that is directly mutagenic for mesothelial cells. By triggering chronic inflammation, frustrated phagocytosis by macrophages also contributes to MPM. Once MPM is diagnosed, the median survival of patients with MPM is about $5-17$ months $(2,3)$. Despite limitations of asbestos usage in industrialized countries, incidence of MPM is nevertheless predicted to peak in the next decade, mainly because of long latency periods preceding neoplasia. Currently available treatments are disappointing: the standard chemotherapy based on an antifolate (pemetrexed) and a DNA-cross-linking agent (cisplatin) only slightly prolongs overall survival (4). Among histological types of MPM (epithelial, sarcomatoid, and biphasic or mixed), the sarcomatoid phenotype is particularly associated with a more aggressive progression. Although imperfectly understood, onset of MPM correlates with modifications in specific genomic regions corresponding to the NF2, TP53, SETD2, and BAP1 genes (5). Among these, loss of BAP1 promotes MPM cell proliferation by upregulating enhancer of zeste homolog 2 (EZH2), the H3 lysine 27-specific (H3K27-specific) N-methyltransferase subunit of the polycomb repressive complex 2 (6). Consistently, proliferation induced by Bap1 deletion is rescued by the loss of EZH2. Based on this concept, S-adenosyl-L-methionine-competitive pharmacological 
inhibitors of EZH2 recently entered clinical trials of advanced solid tumors, B cell lymphomas, and multiple myeloma (https://clinicaltrials.gov: NCT01897571, NCT02395601, NCT02082977, NCT02860286). The preliminary results of these trials seem promising although still unsatisfactory (7). In relapsed or refractory MPM with BAP1 inactivation, a significant proportion of patients achieved disease control in response to tazemetostat monotherapy (i.e., $25 \%$ at 24 weeks) (7).

A major issue is that pharmacological inhibitors of EZH2 not only target the tumor cells, but also indirectly affect the tumor microenvironment. More specifically, macrophages are major modulators of the local antitumor immune response $(8,9)$. In fact, macrophages show a wide range of activation states between the 2 M1 and M2 extremes (i.e., classically and alternatively activated macrophages), depending on stimuli produced in their microenvironment (10). M1 macrophages are induced by TLR-4 agonists released by MPM cells (e.g., HMGB1, HSP90) and proinflammatory cytokines (e.g., IL-12, IFN- $\gamma$ ) and support antitumor immunity. In contrast, M2 and tumor-associated macrophages are induced by antiinflammatory mediators (e.g., IL-10, PGE-2, TGF- $\beta$ ) and promote tumor progression and resistance to therapies (11). Adequate modulation of intratumor macrophages is necessary to support development of cytotoxic T cells' responses toward tumor-associated antigens. Much less understood is the unexpected role of macrophages in direct tumor regression (12). Tumoricidal macrophages can indeed manifest cancer immunoediting activity in the absence of adaptive immunity (13). This concept of cancer immunoediting integrates the dual role of the immune system: host protective (e.g., suppression of tumor growth) and tumor promoting (selection of fitter tumor cells within their microenvironment) (14).

In this report, we address the mechanism of cancer immunoediting in the context of a syngeneic model that includes MPM tumor cells (AB1 and AB12), RAW264.7 macrophages, and BALB/c mice. We demonstrate that macrophages are directly cytotoxic by cell-to-cell contact with MPM cells. Besides ROS and peroxynitrites, EZH2 methyltransferase activity is primarily required for macrophage cytotoxicity toward syngeneic MPM cells. In $2 \mathrm{BALB} / \mathrm{c}$ models injected with sarcomatoid (AB1) and biphasic (AB12) cells, EZH2 inhibition reverts RAW264.7 macrophage-directed tumor regression. Inhibition of macrophage cytotoxicity by EZH2 involves engagement of the PD-1 immune checkpoint.

\section{Results}

Conditioned supernatant from RAW264. 7 macrophages is cytotoxic to AB1 mesothelioma cells. Besides their phagocytic activity, activated macrophages also secrete metabolites that are cytotoxic for tumor cells (15). To address this mechanism in MPM, we stimulated RAW264.7 macrophages with mock agent or LPS for 24 hours, added their culture supernatant (SN) to murine mesothelioma AB1 cells, and measured apoptosis 48 hours later according to the experimental setting of Figure 1A. The representative dot plot shows that, in these conditions, AB1 cells stained positive for propidium iodide and annexin V labeling, indicating onset of apoptosis (compare without SN, SN-mock, and SN-LPS in Figure 1B). As a control, the tyrosine kinase inhibitor lapatinib induced similar rates of apoptosis. Compilation of 8 independent experiments validated this conclusion statistically (Figure 1C). In this model, RAW264.7 cells thus secrete metabolites that are cytotoxic to AB1 MPM cells.

To identify the mechanisms involved in indirect cytotoxicity, we used a pharmacological approach to interfere with NOS, ROS, and peroxynitrites. Synthesis of NO by NOS can be inhibited by an ana$\log$ of L-arginine, L-NMMA. ROS are reduced by apocynin that inhibits the intracellular translocation of 2 critical cytosolic components of the NADPH oxidase complex. Dose response analyses indicated that $1 \mathrm{mM}$ L-NMMA and $300 \mu \mathrm{M}$ apocynin did not affect viability of RAW264.7 and AB1 cells (Supplemental Figure 1; supplemental material available online with this article; https://doi.org/10.1172/jci. insight.128474DS1). L-NMMA at $1 \mathrm{mM}$ significantly reduced the concentration of nitrites in the SN in the presence or absence of LPS (Figure 1D). In these conditions, the cytotoxic activity of RAW264.7-conditioned SN was, however, only marginally affected (Figure 1C). In contrast, inhibition of ROS with a subtoxic dose of $300 \mu \mathrm{M}$ apocynin (Figure 1E) significantly reduced SN cytotoxicity in the presence of LPS (Figure 1C). Similarly, peroxynitrite decomposition by FeTTPS reduced apoptosis of AB1 cells in the presence of LPS (Figure 1C).

These observations thus indicate that ROS and peroxynitrites, but not NO, modulate indirect cytotoxicity of RAW264.7-conditioned SN toward AB1 cells. Because peroxynitrites are formed by the association of NO and superoxide, it appears that ROS are key mediators of cytotoxicity.

RAW264. 7 macrophages are directly cytotoxic for mesothelioma cells by cell-to-cell contact. We next evaluated 
A

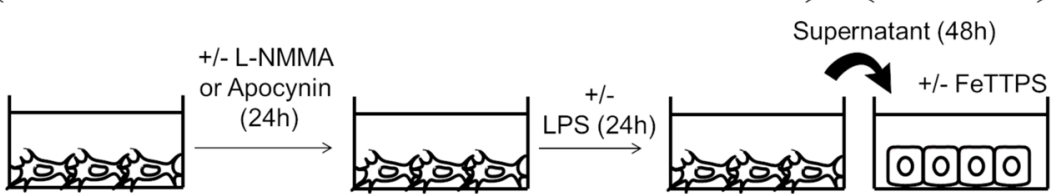

B

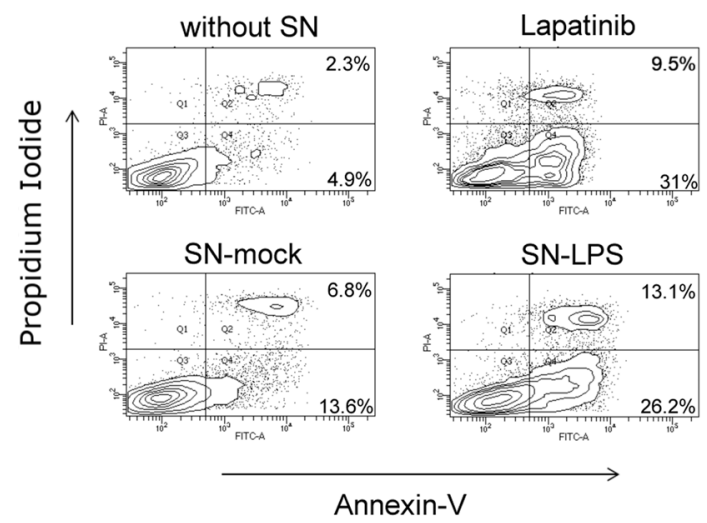

C

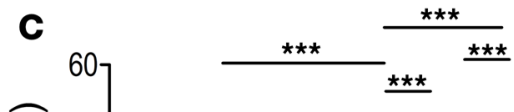

D

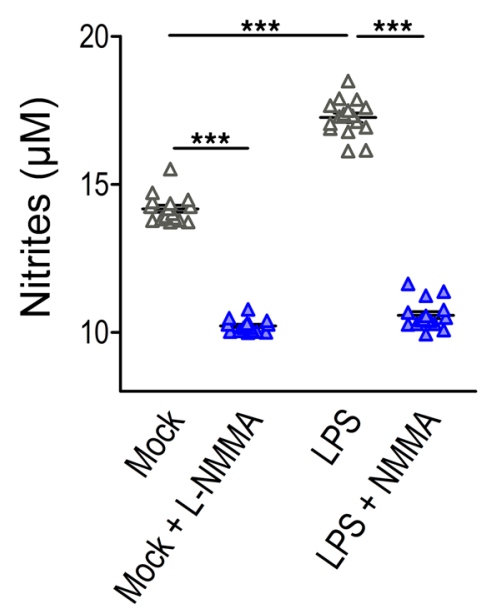

E

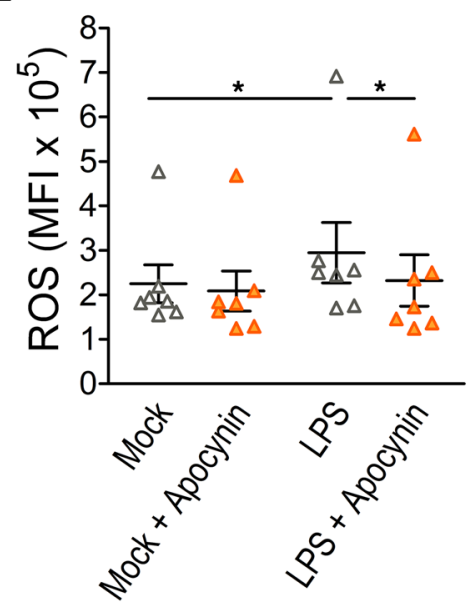

Figure 1. Cytotoxicity of RAW264.7-conditioned SN toward AB1 mesothelioma cells. (A) Experimental design of RAW264.7 macrophages treated with mock agent, $\mathrm{N}^{\mathrm{G}}$-monomethyl-L-arginine (L-NMMA), or apocynin for 24 hours in a 24-well plate. After stimulation with LPS for 24 hours, the cell culture SN was collected and clarified by centrifugation for 6 minutes at $500 \mathrm{~g}$. AB1 cells were cultivated for 48 hours in $25 \%$ macrophage-conditioned SN mixed with 75\% complete RPMI 1640 containing 10\% FCS and 1\% penicillin and streptavidin. The peroxynitrite scavenger [5,10,15,20-tetrakis(4-sulfonatophenyl)-prophyrinato iron (III) chloride; FeTTPS] was directly added to the culture medium. (B) Apoptosis was evaluated after labeling with annexin V-FITC (Becton Dickinson) and propidium iodide (PI, MilliporeSigma). Data were collected with an FACSAria cytometer and analyzed by the FACSDiva software. Treatment with a tyrosine kinase inhibitor (10 $\mu$ M of lapatinib) for 24 hours was used as a positive control. Both $\mathrm{Pl}^{-}$annexin $\mathrm{V}^{+}$and $\mathrm{Pl}^{+}$annexin $\mathrm{V}^{+}$cells were considered to have undergone apoptosis. (C) Apoptotic rates of $\mathrm{AB} 1$ cells were quantified by flow cytometry after labeling with annexin V-FITC. Mean values and standard deviations were deduced from 8 independent experiments. (D) Nitrites $(\mu \mathrm{M})$ were quantified in the cell SN using the Griess reaction assay. (E) Intracellular ROS were measured by flow cytometry using the cell-permeant 2', $7^{\prime}$-dichlorodihydrofluorescein diacetate $\left(\mathrm{H}_{2} \mathrm{DCFDA}\right)$ probe. Each bar represents the mean \pm SEM from 8 independent experiments performed in triplicate. Statistical significance was evaluated using 1-way ANOVA followed by Tukey's multiple-comparisons test. ${ }^{*} P<0.05 ;{ }^{* *} P<0.01$; ${ }^{* * *} P<0.001$. $\mathrm{MFI}$, mean fluorescence intensity. 
the ability of RAW264.7 to kill AB1 mesothelioma cells upon direct contact (Figure 2A). In this system, AB1 cells were not phagocytosed but rather directly killed by LPS-stimulated RAW264.7 macrophages (Figure 2B and Supplemental Videos 1 and 2). Morphological changes associated with AB1 cell death were confirmed by permeability to propidium iodide (Supplemental Figure 2). Confocal microscopy showed that tyrosine nitration to 3-nitrotyrosine, a central modification associated with oxidative stress, occurred in LPS-stimulated, F4/80-labeled RAW264.7 macrophages (N-Tyr in Figure 2C). Upon contact with RAW264.7, AB1 cells also stained positive for 3-nitrotyrosine, revealing macrophage-associated oxidative stress in membrane and cytosolic compartments. Pretreatment of macrophages with L-NMMA only modestly affected AB1 apoptosis in the presence or absence of LPS (Figure 2D). In contrast, inhibition of ROS with apocynin impaired the cytotoxicity of RAW264.7 toward AB1 cells (Figure 2D). To investigate whether ROS-mediated cell death of AB1 cells involved oxeiptosis, phosphoglycerate mutase 5 (PGAM5) expression was silenced by RNA interference using lentiviral transduction of 2 shRNAs (shPGAM\#2 and shPGAM\#5). The levels of PGAM5 transcripts were reduced in AB1 transduced with the PGAM5 shRNAs compared with the scramble control (Figure 2E). When PGAM5 was knocked down, RAW264.7 cell-induced apoptosis of AB1 cells was significantly reduced (Figure 2F).

We conclude that RAW264.7 macrophages are directly cytotoxic to AB1 macrophages through a PGAM5-dependent mechanism that involves oxidative stress.

Pharmacological inhibition of EZH2 reduces direct and indirect cytotoxicity of RAW264. 7 macrophages. Emerging evidence indicates an important role for epigenetic mechanisms in modulating and transmitting signals during macrophage differentiation and polarization (16). Because epigenetic marks such as H3K27me3 orchestrate the magnitude and specificity of gene expression and determine macrophage phenotype, we evaluated the effect of an inhibitor of EZH2 on macrophage cytotoxicity.

RAW264.7 macrophages were incubated with a subtoxic dose of EPZ5687 (10 $\mu \mathrm{M})$, an EZH2 inhibitor (EPZ) for 48 hours and then treated with LPS for an additional day (see Supplemental Figure 1 for dose response of RAW264.7 to EPZ). At this concentration, EPZ did not affect phagocytosis by RAW267.4 (Supplemental Figure 3). As expected, H3K27me3 labeling was reduced by EPZ in confocal microscopy experiments (illustrated in Figure 3A and normalized to Draq5 nuclear staining in Figure 3B). Consistently, flow cytometry confirmed a reduction of $\mathrm{H} 3 \mathrm{~K} 27 \mathrm{me} 3$ fluorescence normalized to total histone H3 (Figure 3C). EPZ only modestly increased the concentrations of ROS and nitrites (Figure 3, D and E). In these experimental conditions, EPZ significantly inhibited apoptosis induced by RAW264.7-conditioned SNs (Figure 3F) and direct cytotoxicity (Figure 3G). Time-lapse microscopy revealed that CFSE-labeled macrophages induced annexin $\mathrm{V}$ staining of $\mathrm{AB} 1$ cells after to cell-to-cell contact, indicating onset of apoptosis (Figure $3 \mathrm{H}$ and Supplemental Video 3). Kinetic analysis confirmed that cocultivation of AB1 cells with RAW264.7 increased the number of annexin $\mathrm{V}^{+} \mathrm{AB} 1$ cells (compare black and gray lines in Figure 3I). Preincubation of macrophages further increased apoptosis (blue line). In the presence of EPZ, the ability of RAW264.7 macrophages to kill AB1 cells was drastically affected (red and green lines in Figure 3I). To further support the involvement of EZH2, RAW264.7 macrophages were transduced with lentiviruses encoding shRNAs. Apoptosis of AB1 cells was significantly reduced when EZH2 was knocked down in RAW264.7 macrophages compared with the scramble control (Supplemental Figure 4).

Together, these data thus show that inhibition of EZH2 reduces direct and indirect cytotoxicity of RAW264.7 macrophages.

Targeting EZH2 abrogates tumor clearance by RAW264.7 macrophages. Because AB1 and RAW264.7 cells were generated on a BALB/c genetic background, we were able to investigate mesothelioma progression in a murine syngeneic system. We used 2 immunocompetent models based on AB1 and AB12 cells pertaining to different subtypes (sarcomatoid and biphasic, respectively) that closely mimic development of human mesothelioma (17). To parallel in vitro studies (Figure 3), RAW264.7 macrophages were cultivated in the presence or absence of EPZ and stimulated or not with LPS. After 3 washes, RAW264.7 cells were inoculated subcutaneously (SC) into the flanks of BALB/c mice with AB1 or $\mathrm{AB} 12$ cells at a 1:3 ratio (Figure 4A). Both mesothelioma cell lines rapidly induced tumor growth (Figure 4, B, D, F, and H) and affected mice viability (Figure 4, C, E, G, and I). RAW264.7 macrophages were not tumorigenic but almost completely abrogated tumor development of AB1 or AB12 cells (Figure 4, B-D). This mechanism was nevertheless ineffective when RAW264.7 macrophages were pretreated with EPZ in culture before inoculation. A similar trend was observed in AB1 sarcomatoid tumors coinjected with RAW264.7 macrophages conditioned in vitro with LPS (compare blue and green lines 
A

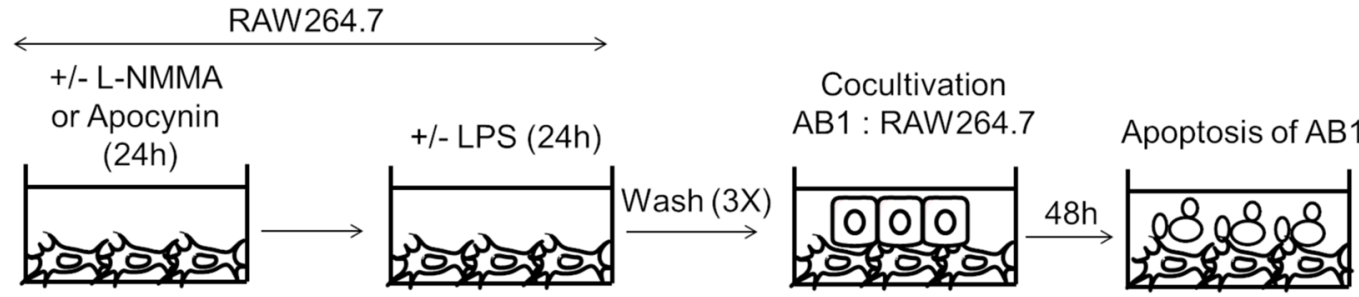

B
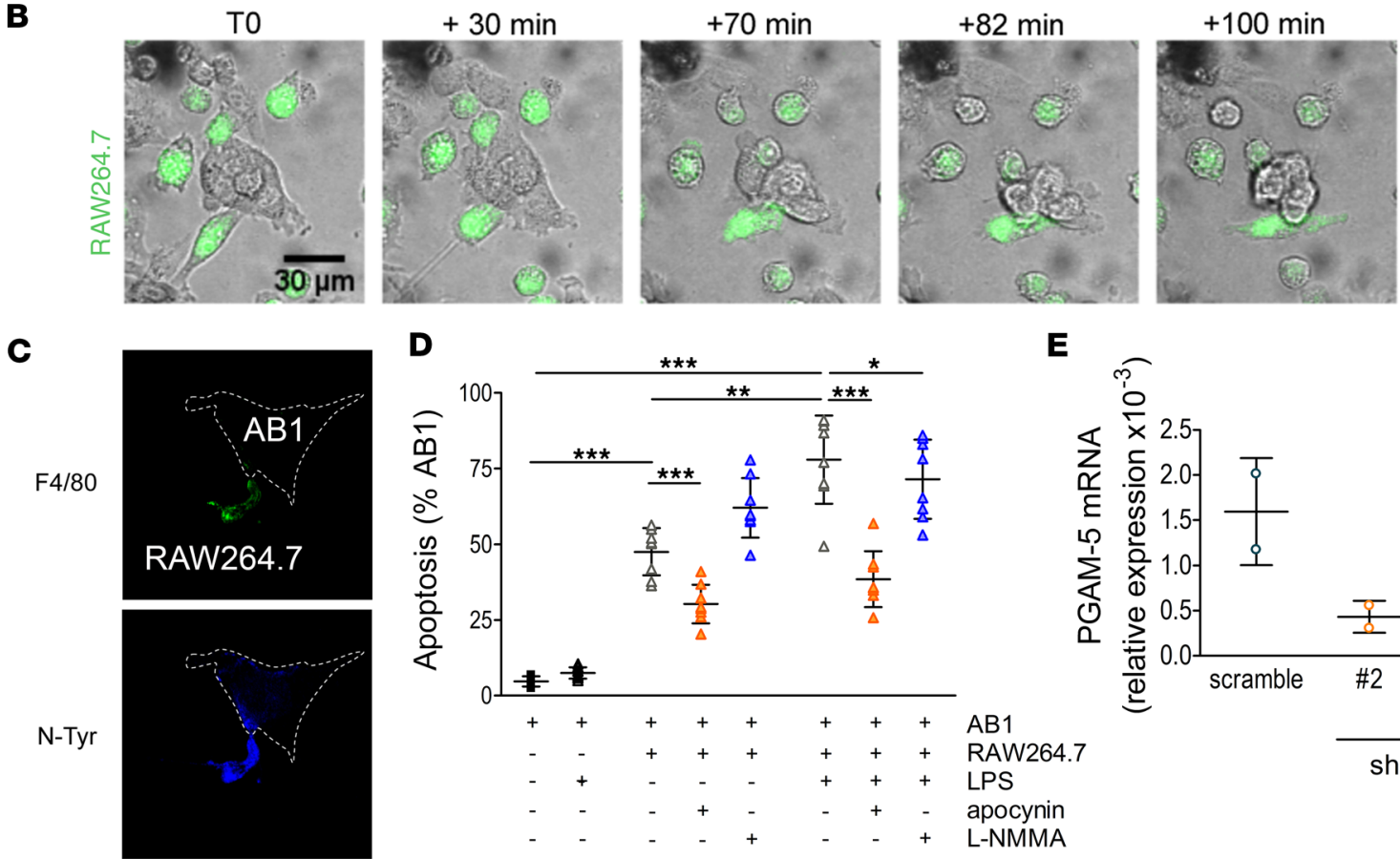

$\mathbf{E}$

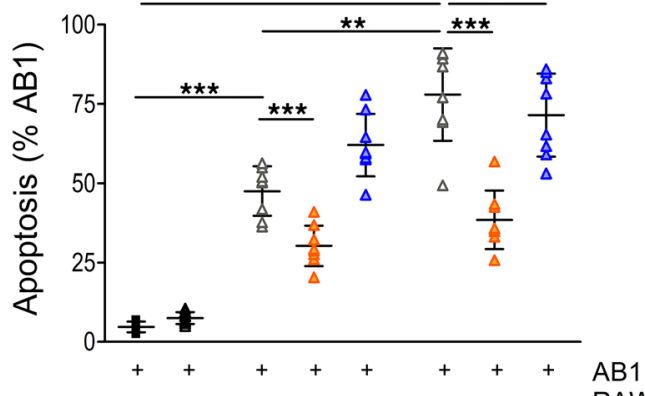

ํㅜㅇ
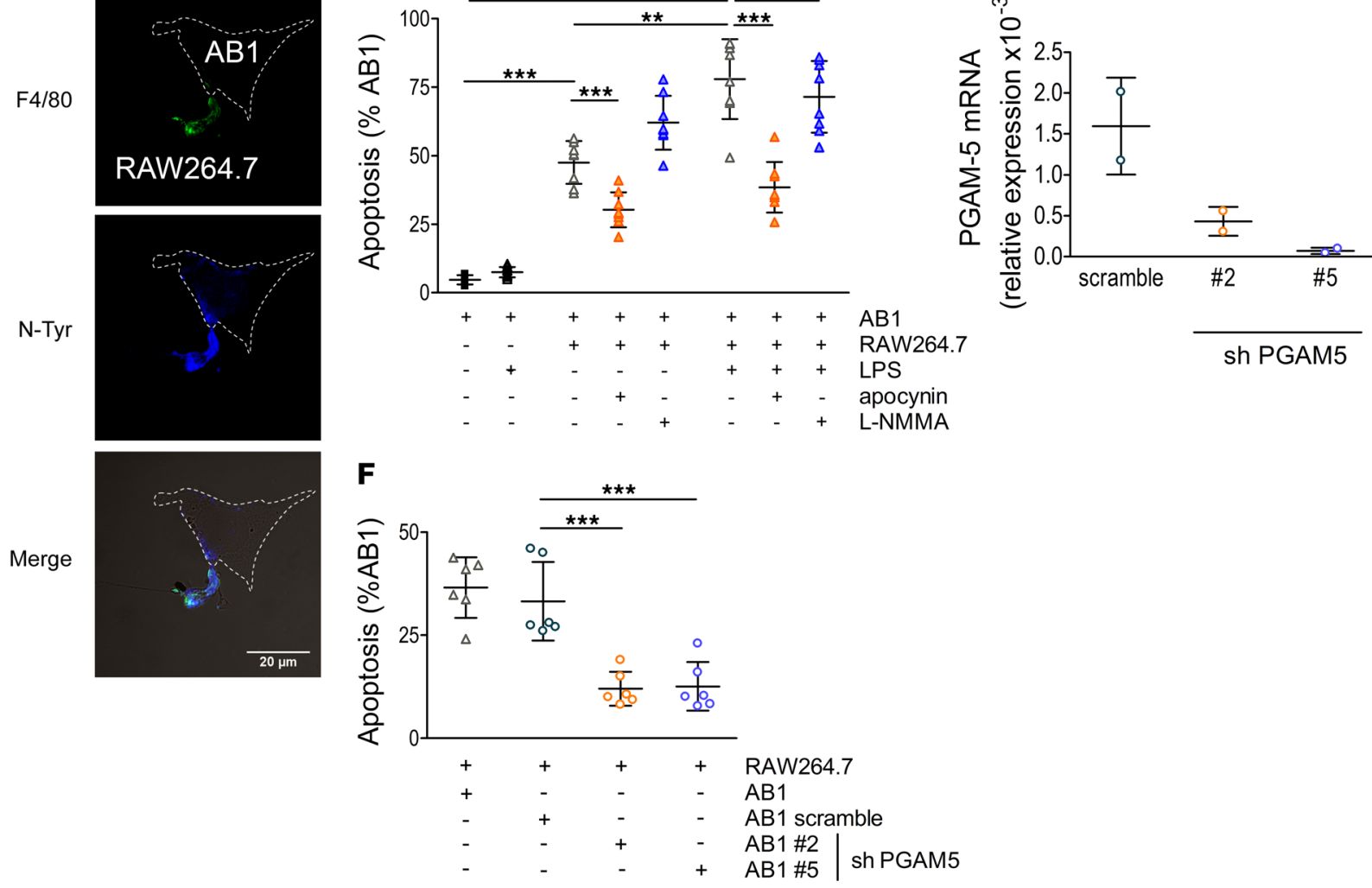

Figure 2. Direct cytotoxicity of RAW264.7 macrophages upon cell-to-cell contact with syngeneic mesothelioma AB1 cells. (A) Experimental design. RAW264.7 macrophages were treated with L-NMMA or apocynin for 24 hours and then further cultivated in the presence or absence of LPS for 24 hours. After 3 washes in PBS, RAW264.7 macrophages were cocultivated with AB1 cells at a 10:1 ratio for 48 hours. (B) AB1 cells and CFSE-labeled RAW264.7 macrophages were monitored by time-lapse microscopy using an LSM 510 (Zeiss) equipped with an environmental chamber maintained at $37^{\circ} \mathrm{C}$ in a humidified $5 \% \mathrm{CO}_{2}$ atmosphere. (C) Cells were fixed, permeabilized, and stained for $\mathrm{F} 4 / 80$ (shown in green) and nitrosylated tyrosine (N-Tyr; shown in blue). Images were acquired using a Zeiss LSM 510 confocal microscope equipped with a $\times 63-1.4$ oil immersion objective. (D) Apoptotic rates of AB1 cells were determined by flow cytometry after staining with the annexin V-FITC kit (Becton Dickinson). Each bar represents the mean \pm SD from 8 independent experiments performed in triplicate. (E) AB1 cells were transduced by lentivectors encoding PGAM5 shRNAs (\#2 and \#5) or a scramble control. The levels of PGAM5 transcripts were measured by reverse transcription quantitative PCR. (F) RAW264.7-induced apoptosis of shRNA-transduced AB1 cells was measured as described in $\mathbf{D}$. Each bar represents the mean \pm SD from 6 independent experiments. Statistical significance was evaluated using 1-way ANOVA followed by Tukey's multiple-comparisons test. ${ }^{*} P<0.05$; ${ }^{* *} P<0.01$; ${ }^{* * *} P<0.001$. 
A

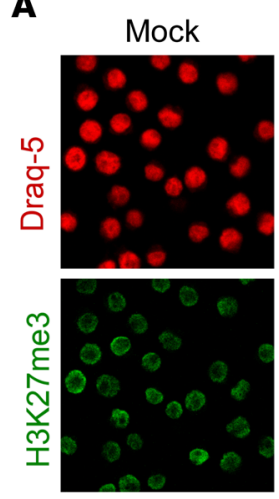

E

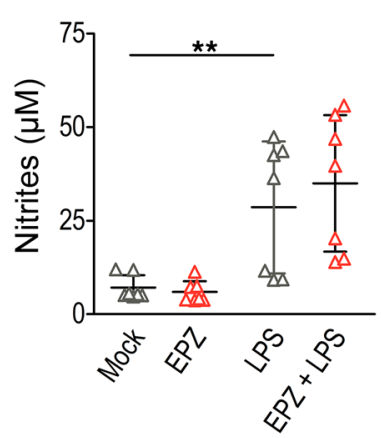

H

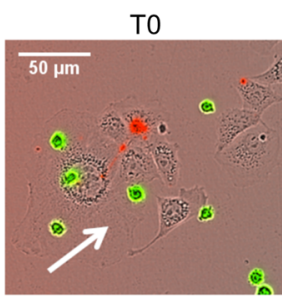

RAW264.7/Annexin-V

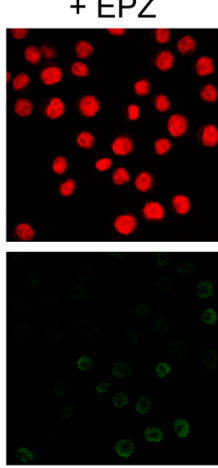

F

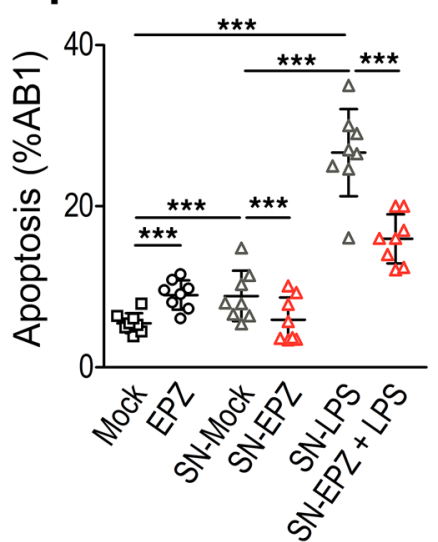

B

C
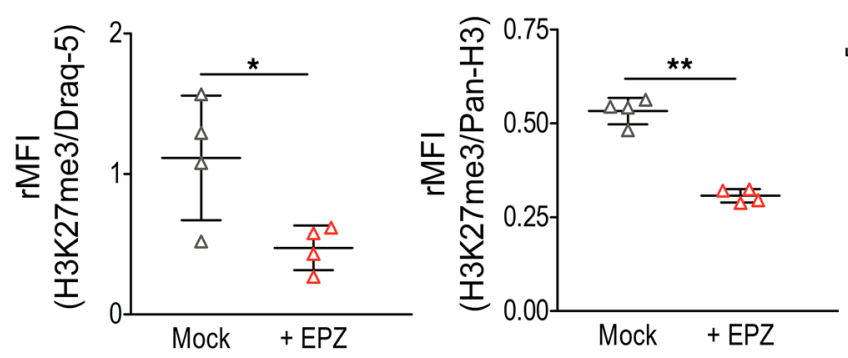

D

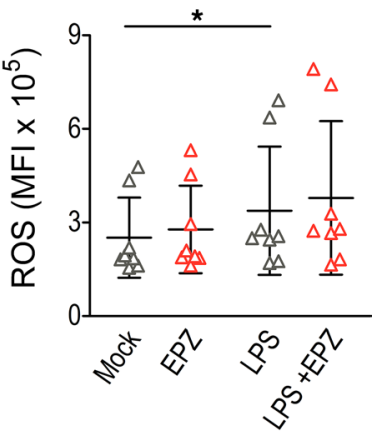

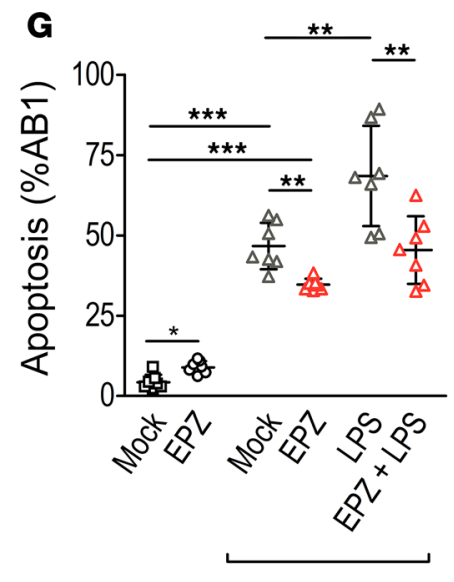

RAW264.7 + AB1

I

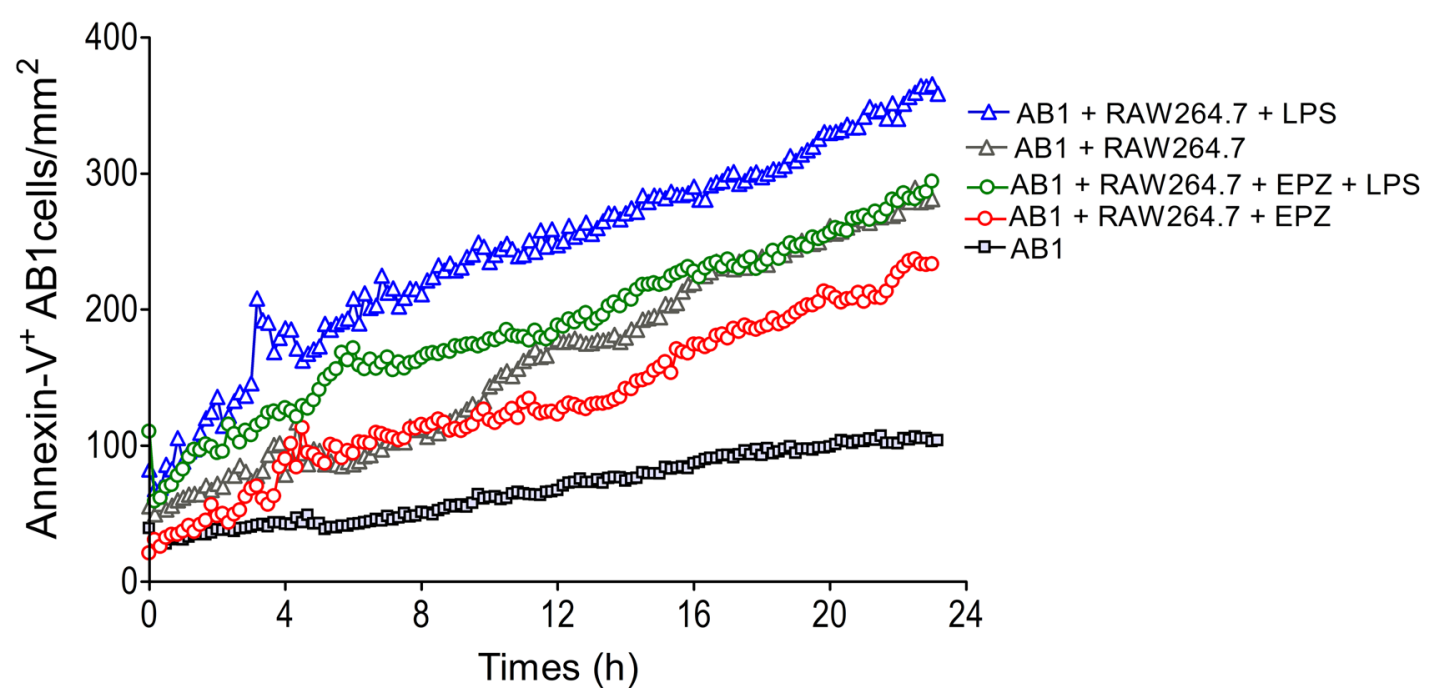


Figure 3. Effect of EZH2 inhibition by EPZ on RAW264.7-mediated killing of AB1 cells. RAW264.7 macrophages were incubated with the EZH2 inhibitor (10 $\mu \mathrm{M}$ EPZ5687) for 48 hours and treated or not with LPS for 24 hours. (A) RAW264.7 macrophages were labeled with an anti-H3K27me3 antibody, stained with Draq5, and analyzed by confocal microscopy. Magnification 40x. (B) The rMFI corresponds to the ratio of fluorescence intensities associated with H3K27me3 and Draq5. (C) RAW264.7 macrophages were fluorescently labeled with antibodies against pan-Histone 3 or H3K27me3 and analyzed by flow cytometry using a FACSaria flow cytometer (Becton Dickinson). The rMFI represents the ratio of fluorescence intensities associated with H3K27me3 and pan-H3. (D) Intracellular ROS were measured by flow cytometry using the cell-permeant $\mathrm{H}_{2} \mathrm{DCFDA}$ probe. MFI, mean fluorescence intensity. (E) Nitrites (in $\mu \mathrm{M}$ ) in the cell SNs were quantified by the Griess reaction assay. (F) RAW264.7 macrophage-conditioned SNs were added to $A B 1$ cell cultures as described in Figure $1 A$. Apoptosis was determined by flow cytometry after labeling with annexin V-FITC. (G) CFSE-labeled RAW264.7 macrophages were cocultivated for 48 hours with AB1 cells at a 10:1 ratio. Apoptotic rates of CFSE- AB1 cells were determined by flow cytometry after staining with the annexin V-FITC kit (Becton Dickinson). Each bar represents the mean \pm SD. Statistical significance was evaluated using the paired Student's $t$ test (B and $\mathbf{C}$ ) and 1-way ANOVA followed by Tukey's multiple-comparisons test $(\mathbf{D}-\mathbf{G})$. ${ }^{*} P<0.05$; ${ }^{* *} P<0.01 ;{ }^{* * *} P<0.001$. (H) CFSE-labeled RAW264.7 macrophages were cocultivated for 24 hours with AB1 cells at a 1:1 ratio in the presence of annexin V-APC (red fluorescence). The cells were monitored by the IncuCyte S3 Live-Cell imaging system (Essen Bioscience) placed in an incubator maintained at $37^{\circ} \mathrm{C}$ in a humidified 5\% $\mathrm{CO}_{2}$ atmosphere. (I) RAW264.7 macrophages pretreated with EPZ and/or LPS were cocultured with CFSE-labeled AB1 cells. The number of $\mathrm{CFSE}^{+}(\mathrm{AB} 1)$ annexin $\mathrm{V}^{+}$events was determined every 10 minutes for 24 hours using the IncuCyte $\mathrm{S} 3$ Live-Cell imaging system. Each curve is the average of 5 independent experiments performed in triplicate.

in Figure 4F). Pretreatment of RAW264.7 macrophages with LPS abolished their ability to control tumor growth in biphasic AB12 cells (Figure 4H).

Hematoxylin and eosin counterstaining revealed infiltration of immune cells, such as macrophages, granulocytes, and lymphocytes, in RAW264.7 AB1 tumors (Figure 5A). When RAW264.7 macrophages were coinjected with $\mathrm{AB} 1$ cells, infiltration of $\mathrm{CD}^{+}$cells increased (Supplemental Figure 5A). In contrast, $\mathrm{AB} 1$ tumors of similar size displayed a different pattern of fusiform cells. Tumors were analyzed by confocal microscopy using macrophage-specific monoclonal F4/80 and antibodies against cleaved caspase- 3 . Figure 5B illustrates pseudopodia from macrophages interacting with apoptotic neighboring mesothelioma cells. Quantification of cleaved caspase-3-associated fluorescence revealed that apoptosis was significantly reduced in AB1 tumors coinjected with EPZ-treated RAW264.7 cells (Figure 5C).

We conclude that (a) RAW264.7 cells efficiently control tumor development in 2 immunocompetent models of murine mesothelioma and (b) EZH2 is required for macrophage cytotoxicity.

Inhibition of EZH2 stimulates PD-1 expression on RAW264.7 while PD-1 blockade restores macrophage cytotoxicity. Because PD-1 has been associated with inhibition of phagocytic potency (18), we hypothesized that a similar mechanism also operated in macrophage-directed cytotoxicity. PD-1 expression in tumor biopsies increased when AB1 cells were coinjected with RAW264.7 macrophages compared with AB1 alone (Supplemental Figure 5B). When RAW264.7 cells were pretreated with LPS or EPZ, PD-1 expression was further stimulated in tumors. Flow cytometry demonstrated that EPZ enhanced PD-1 expression in the presence and absence of LPS (Figure 6, A and B). In the presence of EPZ, PD-1 expression increased at both the protein and RNA levels and was associated with a reduction of H3K27me3 at the PD-1 promoter (Supplemental Figure 6). Time-lapse analysis showed that PD-1 (stained in red on Figure 6C) localized at the synapse between RAW264.7 and AB1 (arrows at T0 $+2 \mathrm{~h}$ in Figure 6C). Engagement of PD-1 between EPZ-conditioned macrophages and AB1 cells resulted in killing inhibition (Supplemental Video 4). Blockade with an anti-PD-1 antibody but not with an isotype control restored apoptosis of AB1 cells (Figure 6D). Blockade of the PD-1 ligand (PD-L1) resulted in an intermediate effect compared with the isotype control (Supplemental Figure 7). In the mouse model, macrophages pretreated with EPZ and anti-PD-1 antibody efficiently controlled AB1 and AB12 tumor growth (Figure 6, E and F, and Supplemental Figure 8).

Together, these data demonstrate that EPZ stimulates expression of the PD-1 checkpoint inhibitor on RAW264.7 macrophages. Blockade of PD-1 restores the antitumor activity of EPZ-treated macrophages toward AB1 cells.

\section{Discussion}

In MPM, macrophages are massively recruited into the pleura and orchestrate an early inflammatory response toward tumor cells $(9,19,20)$. This antitumor immunity is initiated by a series of features that distinguish normal from mesothelioma cells (e.g., altered expression of genes involved in cell metabolism and apoptosis). Tumor cells also release danger-associated molecular patterns that are detected by the innate immune system (21, 22). Mesothelioma cells further secrete endogenous ligands of TLR-4, such as HMGB-1, tenascin-C, and HSP-70/90 (23-28). Upon initiation, macrophages phagocytose and degrade cancer cells $(29,30)$. In 
A
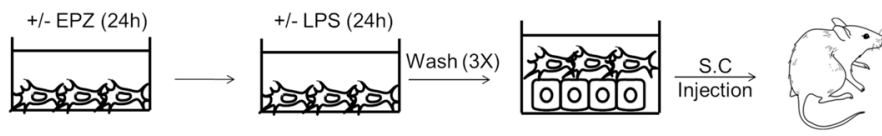

B

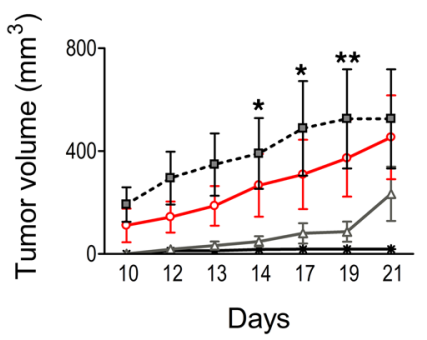

D

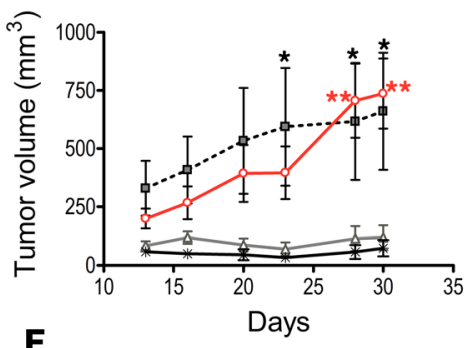

\section{$\mathbf{F}$}
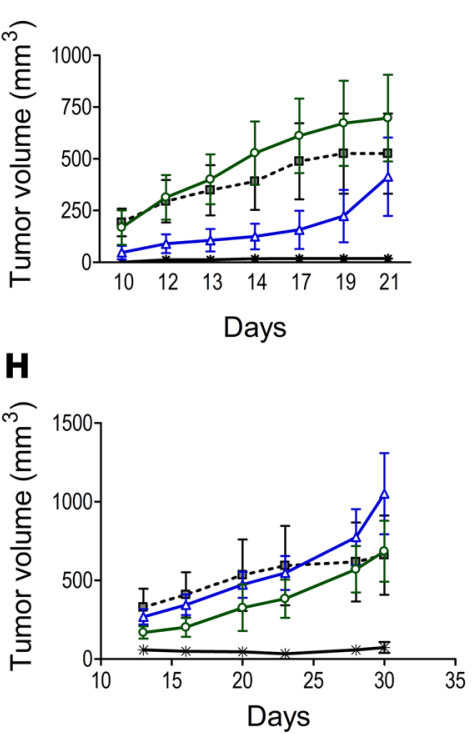

C

BALB/c mice

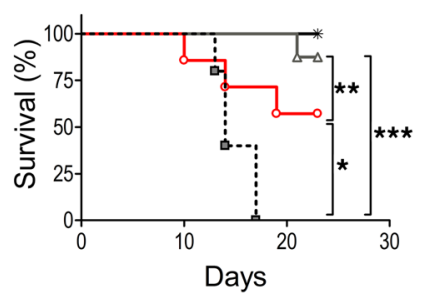

E

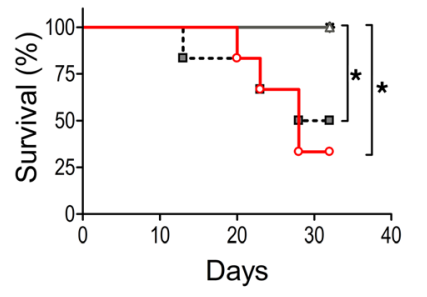

G

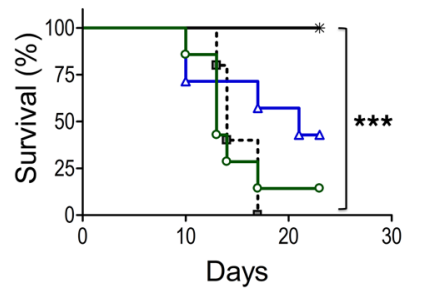

I

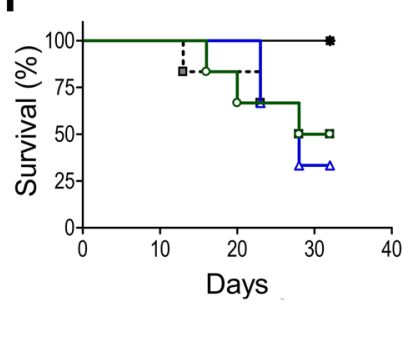

- $-\mathrm{AB} 1$

-RAW264.7

$\triangle-\mathrm{AB} 1+\mathrm{RAW} 264.7$

$-a-A B 1+R A W 264.7+E P Z$

- $-A B 12$

*-RAW264.7

$-\triangle A B 12+R A W 264.7$

- $-\mathrm{AB} 12+\mathrm{RAW} 264.7+\mathrm{EPZ}$
-a.AB12

* RAW264.7

$\rightarrow-$ AB12 + RAW264.7 + LPS

$\rightarrow$ AB12 + RAW264.7 + LPS + EPZ

Figure 4. Effect of EPZ on RAW264.7-mediated inhibition of AB1 tumor growth. (A) RAW264.7 macrophages were cultured with the EZH2 inhibitor ( $10 \mu \mathrm{M}$ EPZ5687) for 24 hours and stimulated with LPS for an additional day. After 3 washes, RAW264.7 cells were coimplanted subcutaneously (SC) in BALB/c mice with $2 \times 10^{6} A B 1$ or AB12 cells at a 1:3 ratio. In B, D, $\mathbf{F}$, and $\mathbf{H}$, tumor volumes (in $\mathrm{mm}^{3}$ ) were calculated weekly using the following formula: $4 / 3 \times \pi \times(\text { diameter } / 2)^{3}$. C, E, G, and I represent the corresponding survival curves. Groups of at least 6 mice were tested in each experimental condition. All data are plotted as mean $\pm \operatorname{SEM}(n=6)$. Statistical significance was evaluated using 2-way ANOVA with Bonferroni's post test (B, $\mathbf{D}, \mathbf{F}$, and $\mathbf{H}$ ) and log-rank $\chi^{2}$ test (survival curves). ${ }^{*} P<0.05 ;{ }^{* *} P<0.01 ;{ }^{* *} P<0.001$.

this report, we investigated a different mechanism of macrophage-dependent anticancer activity that does not involve prior phagocytosis. As $\mathrm{CD}^{+}$cytotoxic $\mathrm{T}$ cells, macrophages are indeed well armed to directly destroy tumor cells (e.g., reactive oxygen/nitrogen species [RONS], FasR/FasL, TRAIL-R/TRAIL-L, TNF-R/ TNF- $\alpha$ ). Using 2 mesothelioma cell lines, we demonstrate here that autologous RAW264.7 macrophages direct cytolytic activity before phagocytosis (Figure 2). This activity primarily requires ROS as revealed by the 
A

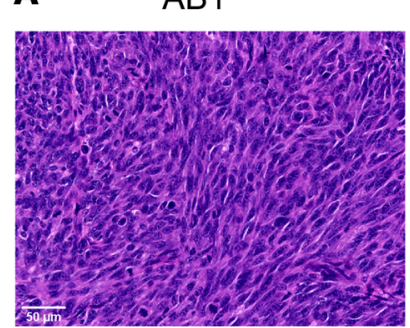

AB1 + RAW264.7

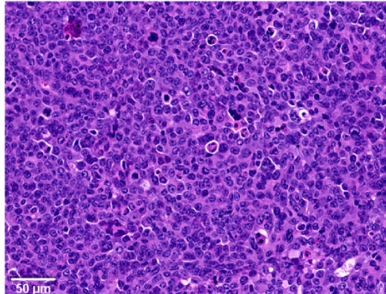

$A B 1+R A W 264.7+E P Z$

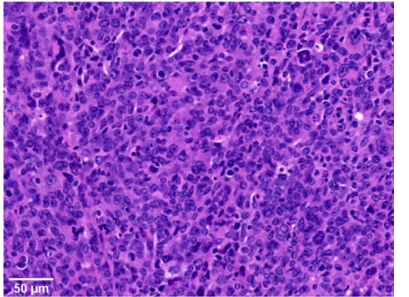

B
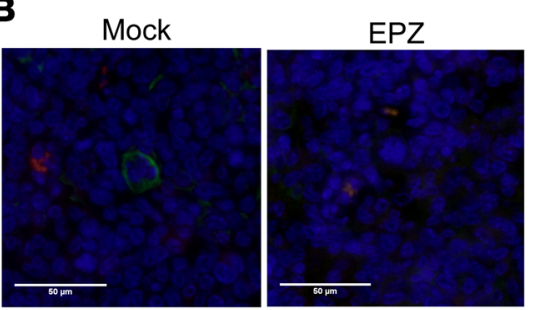

$A B 1+R A W 264.7$

Caspase 3 ; F4/80; DAPI

LPS

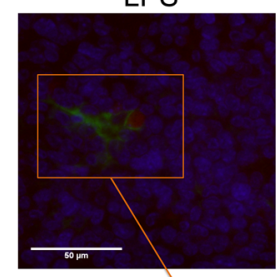

$\checkmark$

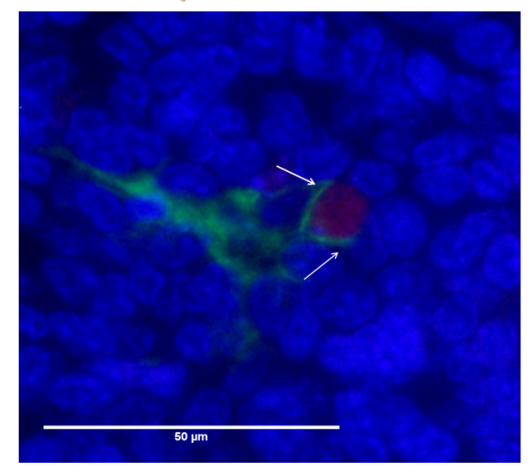

LPS + EPZ

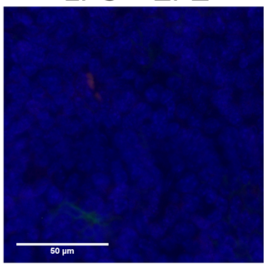

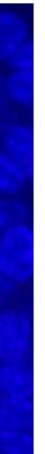

Caspase 3 F/80, DAPI

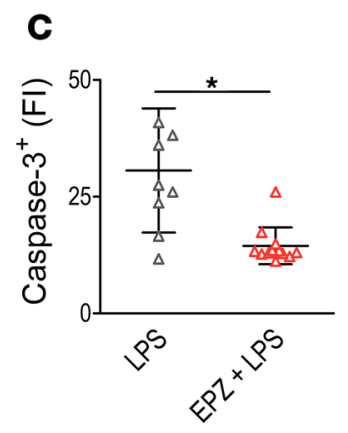

Figure 5. Immunohistochemistry of AB1 mesothelioma tumors. (A) Histochemical analyses of tumors from BALB/C mice inoculated with AB1 tumor cells and RAW264.7 macrophages differentiated in the presence or absence of EPZ and/or LPS. Sections from tumors with similar volumes were stained with hematoxylin and eosin (original magnification, $\times 20$ ). (B) Representative immunohistochemistry micrographs of apoptotic cells and F4/80+ macrophages. Apoptotic cells were labeled with an antibody directed against cleaved caspase-3 (active) and an Alexa Fluor 546 conjugate (in red). Infiltrating macrophages were identified by F4/80 and anti-IgG Alexa Fluor 488 antibodies (in green). Cell nuclei were stained with DAPI (in blue). The higher magnification (original magnification, $\times 40$ ) reveals macrophage pseudopodia interacting with an apoptotic tumor cell (white arrows). (C) The cleaved caspase-3 fluorescence intensity (FI) was quantified from 25 images $(3 \times 3$ segmentation at original magnification $\times 40)$ by using Image and FSX100. Each bar represents the mean $\pm \mathrm{SD}$. ${ }^{*} P<0.05$, evaluated using the 2-tailed Mann-Whitney $U$ test.

use of apocynin, a pharmacological inhibitor of the superoxide radical $\left(\mathrm{O}_{2}^{-}\right)$that interferes with intracellular translocation of the cytosolic component (p47-phox and p67-phox) of NADPH oxidase (31). In contrast, inhibition of NOS by L-NMMA (32) has no effect in our experimental settings likely because NO recombines with the short-lived $\left(\mathrm{O}_{2}^{-}\right)$radical to generate peroxynitrite anion $\left(\mathrm{ONOO}^{-}\right)(33,34)$. Consistently, neutralization of peroxynitrites with FeTTPS quenched the cytotoxic potency of LPS-primed, RAW264.7-conditioned SN (Figure 1C). Note that the involvement of peroxynitrites in direct cytotoxicity could not be determined because FeTTPS excessively reduced RAW264.7 metabolic activity (Supplemental Figure 1). Furthermore, covalent association of $\mathrm{NO}_{2}$ - with the aromatic ring of tyrosine (3-nitrotyrosination) appeared in $\mathrm{AB} 1$ meso- 
A

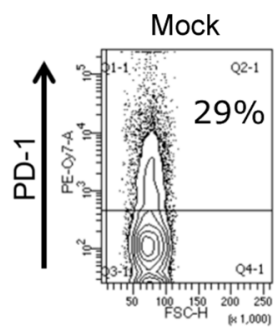

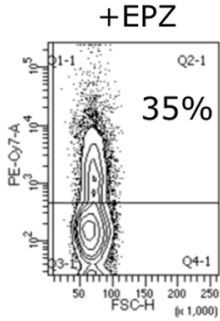

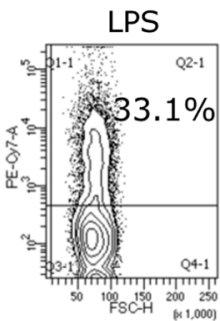

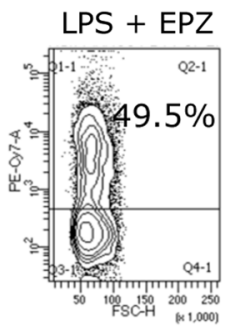

B

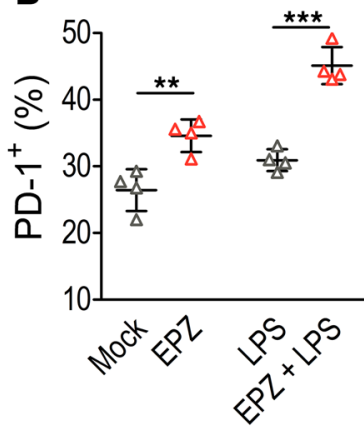

C

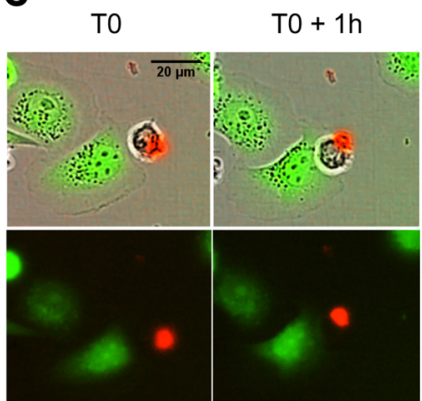

CFSE(AB1)/PD-1
$\mathrm{T} 0+2 \mathrm{~h}$

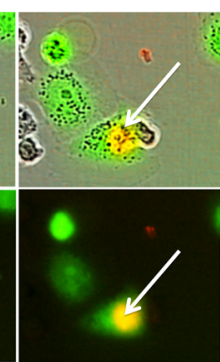

$\mathrm{TO}+3 \mathrm{~h}$

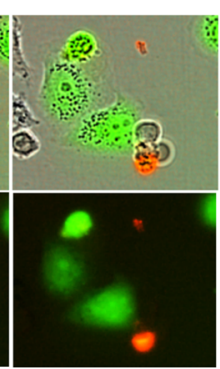

e

E

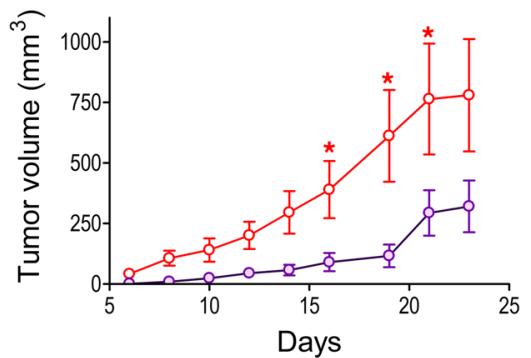

$\mathbf{F}$

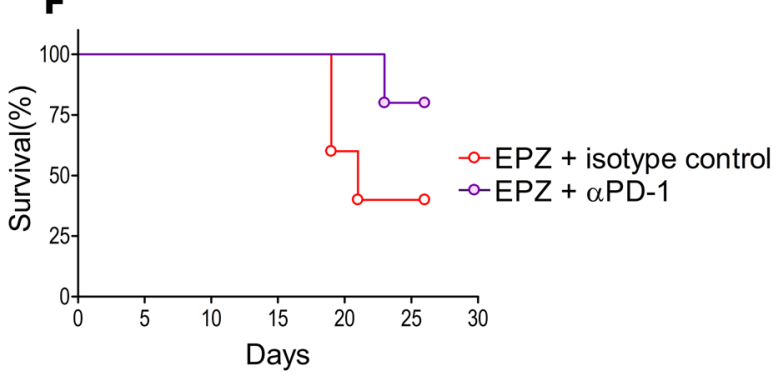

Figure 6. Effect of EPZ on PD-1 expression and activity of anti-PD-1 blockade in AB1/RAW264.7 cocultures. (A) RAW264.7 macrophages were cultured in the presence of LPS and/or EPZ as described in Figure 3. After labeling with an anti-mouse PD-1 antibody, fluorescence emission was analyzed by flow cytometry using a BD FACSAria. Representative dot plots of FSC-H ( $x$ axis) and percentage of PD-1 cells ( $y$ axis) are shown. (B) Percentages of RAW264.7 macrophages expressing PD-1 were deduced from 4 independent experiments. Each bar represents the mean $\pm S D$. ${ }^{* *} P<0.01$, and ${ }^{* * *} P<0.001$, calculated using 1-way ANOVA followed by Tukey's multiple-comparisons test. (C) Time-lapse analysis of CFSE-labeled AB1 cells cocultured with RAW264.7 macrophages for 24 hours at a 1:1 ratio in the presence of PE/Cy7-conjugated anti-mouse PD-1 antibody (red). (D) RAW264.7 macrophages were incubated with anti-PD-1 antibody (10 $\mu \mathrm{g} / \mathrm{mL}$; InVivoMAb, BioXcell) or rat IgG2a isotype control for 6 hours and then cocultivated with CFSE-labeled AB1 cells in the presence of annexin V-APC. The cells were monitored by the IncuCyte S3 Live-Cell imaging system (Essen Bioscience) placed in an incubator maintained at $37^{\circ} \mathrm{C}$ in a humidified $5 \% \mathrm{CO}_{2}$ atmosphere. The number of annexin $\mathrm{V}^{+} \mathrm{AB} 1$ cells (percentage) was quantified every 10 minutes for 24 hours. Statistical significance was evaluated using 2-way ANOVA with Bonferroni's post test. ${ }^{* *} P<0.001$. (E) RAW264.7 macrophages were cultured with the EZH2 inhibitor $(10 \mu \mathrm{M}$ EPZ5687) for 24 hours and with anti-PD-1 antibody (10 $\mu \mathrm{g} / \mathrm{mL}$; InVivoMAb, BioXcell) or Rat IgG2a isotype control (10 $\mu \mathrm{g} / \mathrm{mL}, \mathrm{BD})$ for 6 hours before inoculation. After 3 washes, RAW264.7 macrophages were coimplanted SC into BALB/c mice with $2 \times 10^{6} \mathrm{AB} 1$ or AB12 cells at a 1:3 ratio. Tumor volumes (in $\mathrm{mm}^{3}$ ) were calculated weekly using the following formula: $4 / 3 \times \pi \times(\operatorname{diameter} / 2)^{3}$. (F) The corresponding survival curve for $\mathbf{E}$. Groups of at least 6 mice were tested in each experimental condition. All data are plotted as mean $\pm \operatorname{SEM}(n=6)$. Statistical significance was evaluated using 2 -way ANOVA with Bonferroni's post test $(\mathbf{E})$ and log-rank $\chi^{2}$ test $(\mathbf{F}) .{ }^{*} P<0.05 ;{ }^{* *} P<0.01 ;{ }^{* *} P<0.001$.

thelioma cells upon contact with RAW264.7 (Figure 2C). 3-nitrotyrosination also occurred in the activated RAW264.7 as well as in neighboring cells (data not shown), further supporting the role of RONS and peroxynitrites in macrophage-mediated cell killing. RAW264.7 macrophages are thus similar to dendritic cells that also use peroxynitrite to exert their tumoricidal activity upon activation by LPS $(35,36)$. ROS and peroxynitrites induce oxeiptosis via the Kelch-like ECH-associated protein 1/nuclear factor erythroid 2-related factor 2 (Keap1/Nrf2) pathway (37). This mechanism is involved in cytoprotective response to endogenous and exogenous ROS. Nrf2 is a transcription factor that induces expression of antioxidant enzymes, such as $\mathrm{NAD}(\mathrm{P}) \mathrm{H}$ quinone oxidoreductase 1 , heme oxygenase 1, glutamate-cysteine ligase, and glutathione- $S$-transferases. Keap1 binds Nrf2 and induces its ubiquitin-dependent degradation. Under oxidative stress, Keap1 is 
inactivated by oxidation of cysteine residues, causing Nrf2 accumulation in cells. PGAM5 is pivotal to mediate Keap1-dependent cells' death in response to ROS. Oxidized Keap1 releases PGAM5 to induce caspase-independent cell death. RNA interference data (Figure 2F) show that RAW264.7 macrophages induced AB1 cell death in a PGAM5-dependent manner.

Besides RONS and peroxynitrites, RAW264.7 cytotoxic activity against AB1/AB12 cells also requires EZH2 as revealed by the pharmacological inhibitor EPZ5687. Our preliminary results further show that the cytotoxicity of human macrophages pulsed with pleural effusion from MPM patients was also inhibited by an inhibitor of EZH2 (data not shown). Furthermore, we have also obtained evidence that macrophages are cytotoxic in spheroid cell culture systems of RAW264.7 and AB1/AB12 cells (data not shown).

Evidence obtained in cell culture (Figure 3) was further validated by 2 autologous mouse models of mesothelioma (Figures 4 And 5). Tumor growth of sarcomatoid (AB1) and biphasic (AB12) cells was abrogated by coinjection of RAW264.7 macrophages but restored by pretreatment with the EZH2 inhibitor. Because the inhibitor is washed out before macrophage inoculation, this experimental setting thus investigates RAW264.7 cytotoxicity rather than direct antitumor activity of EPZ. Pretreatment with LPS abolished the capacity of RAW264.7 cells to efficiently control AB1/AB12 tumor growth (Figure 4, F-I) perhaps after LPS-induced inflammation or, as indicated by Supplemental Videos 1 and 4, after macrophage apoptosis or induction of endotoxin tolerance $(18,38-42)$. In fact, the macrophage response to LPS depended on the dose and the duration of stimulation. The chronic or repeated exposure to TLR-4 agonists led to macrophage endotoxin tolerance or macrophage exhaustion. Stimulation with LPS augmented PD-1 mRNA and protein levels in RAW264.7 macrophages (data not shown). Furthermore, the number of PD-1 $1^{+}$cells increased upon LPS pretreatment of RAW264.7 cells (Supplemental Figure 5B). Taken together, these observations suggest that LPS-primed RAW264.7 macrophages show an exhausted phenotype. Figure 6 shows that AB1 mesothelioma cells were able to escape killing by engaging the PD-1 checkpoint on RAW264.7 macrophages.

This conclusion is of paramount importance for clinical trials that currently target EZH2 in different cancers with pharmacological inhibitors, such as tazemetostat. Indeed, EZH2 inhibitors are being evaluated in advanced solid tumors, B cell lymphomas, and multiple myeloma (https://clinicaltrials.gov: NCT01897571, NCT02395601, NCT02082977, NCT02860286). The H3K27me3 epigenetic mark catalyzed by EZH2 is a repressive marker leading to aberrant gene expression during malignant transformation (43). Silenced genes exhibit decreased chromatin accessibility and diminished recruitment of transcription factors, such as p65. Inhibition of EZH2 by RNA interference decreases tumorigenicity of malignant mesothelioma cells (43). Besides this antitumor activity, our report indicates that the EZH2 methyltransferase exerted an important role in modulating and transmitting signals during macrophage differentiation or polarization. In MPM mouse models (Figures 4 And 5), EZH2 was even a limiting factor that was required for antitumor activity associated with direct macrophage cytotoxicity. The EZH2 EPZ inhibitor only modestly affected RONS (Figure 3, D and E) but stimulated PD-1 expression in macrophages (Figure 6). Besides the involvement of PD-1 in phagocytosis (18), engagement of this checkpoint inhibitor may therefore also explain the inability of macrophages to exert cell-to-cell direct cytotoxicity.

In summary (Figure 7), we have shown that RAW264.7 macrophages are cytotoxic to AB1 cells by direct cell-to-cell contact via oxeiptosis. In the absence of prior phagocytosis, RAW264.7 macrophages are thus directly cytotoxic to mesothelioma cells. In autologous mouse models, RAW264.7 macrophages impair tumor growth of $\mathrm{AB} 1 / \mathrm{AB} 12$ mesothelioma cells. This activity is abrogated by an inhibitor of EZH2 that stimulates expression of PD-1 (Figure 7). This conclusion justifies the combination of immune checkpoint and EZH2 inhibitors in clinical trials in mesothelioma as well as in other solid tumors (e.g., NCT03337698). Considering the complex interactions between EZH2 inhibitors, adaptive resistance to tumor immunotherapy, and macrophage direct cytotoxicity (44), understanding the molecular mechanisms involved should be considered to design new therapeutic approaches.

\section{Methods}

Cell culture. RAW264.7 (ATCC, TIB-71), murine monocytic cells isolated from BALB/c mice, were maintained in DMEM supplemented with 10\% heat-inactivated FCS (Lonza), along with penicillin and streptavidin (100 $\mathrm{UI} / \mathrm{mL}$, BioWhittaker). AB1 and AB12 mesothelioma cell lines generated after intraperitoneal injection of $\mathrm{BALB} / \mathrm{c}$ mice with crocidolite asbestos were cultivated in complete RPMI 1640 medium (Lonza) containing $10 \%$ FCS and antibiotics (penicillin and streptomycin). Cell lines were maintained at $37^{\circ} \mathrm{C}$ in a humidified 
Indirect cytotoxicity
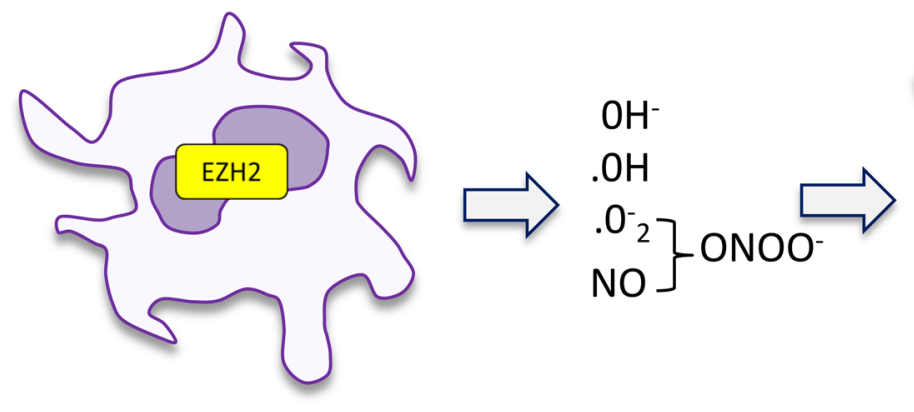

Direct cytotoxicity

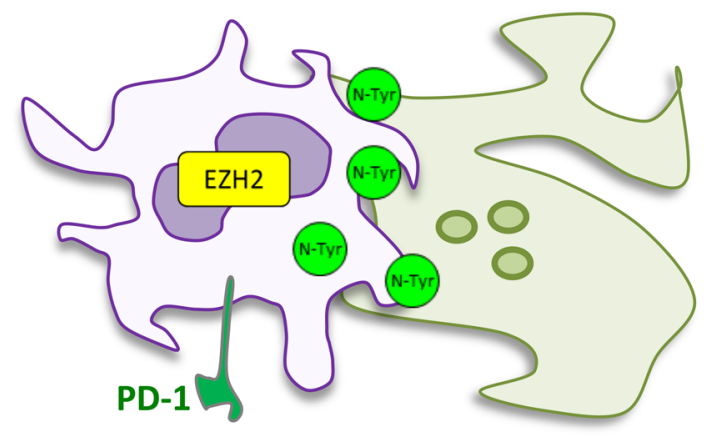

Depletion/anergy

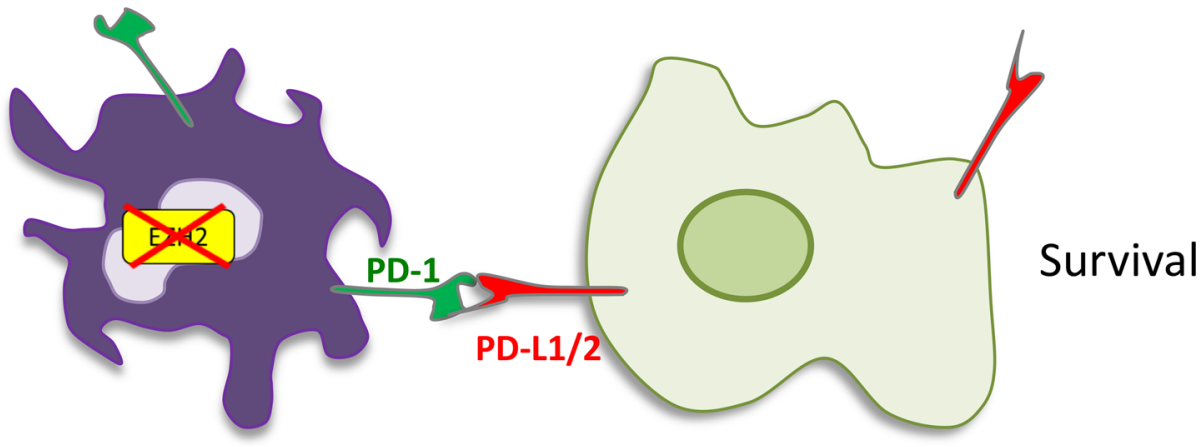

$\sqrt{1}$

Immunotherapy

Anti-PD-1

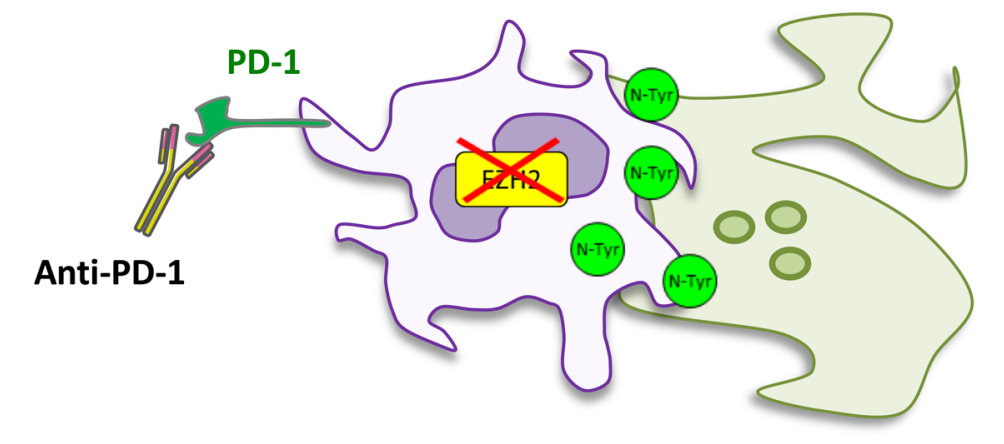

Figure 7. A model for EZH2dependent immunoediting of mesothelioma cells by autologous macrophages. Schematic representation of direct and indirect cytotoxicities mediated by ROS and peroxynitrites produced by macrophages. Oxidative stress induces tyrosine nitration in tumor cells, leading to apoptosis and phagocytosis by macrophages. Inhibition of $\mathrm{EZH} 2$ reduces direct cytotoxicity exerted by macrophages without affecting ROS production but increases PD-1 expression. The engagement of PD-1 at the synapse between macrophages and mesothelioma cells impairs killing activity of macrophages. The combination of an $\mathrm{EZH} 2$ inhibitor (such as tazemetostat) and immune checkpoint inhibitor that targets $\mathrm{PD}-1$ (e.g., pembrolizumab, nivolumab) restores immunoediting activity of macrophages. 
5\% $\mathrm{CO}_{2}$ air atmosphere. RAW264.7 macrophages were transduced with lentiviral vectors (pLVs) coexpressing GFP and shRNA sequences targeting EZH2 (shRNA\#1 5'-TTGAGTACTGTGGGCAATTTA-3', shRNA\#3 5'-ACTTGCCCACCTCGGAAATTT-3') or a scramble sequence (5'-CCTAAGGTTAAGTCGCCCTCG-3') (Vector Builder). AB1 cells were transduced with pLVs coexpressing GFP and shRNA sequences targeting PGAM5 (shRNA\#2 5'-GCCTGGGATTAAAGTTTAATA-3', shRNA\#5 5'-CTGGAGAAGACGAGTTGACAT-3') or a scramble sequence (5'-CCTAAGGTTAAGTCGCCCTCG-3') (Vector Builder). The transduced RAW264.7 and AB1 cells were selected during 3 weeks with G418 (1 mg/mL, Roche).

Indirect cytotoxicity assay. RAW264.7 macrophages were grown in a 24-well plate $\left(5 \times 10^{4}\right.$ cells/well $)$ in the presence of apocynin (300 $\mu \mathrm{M}$, Sigma-Aldrich), L-NMMA (1 mM, Sigma-Aldrich), or EPZ (10 $\mu$ M, Selleck Chemicals) for 48 hours and stimulated by LPS ( $1 \mu \mathrm{g} / \mathrm{mL}$, LPS from E. coli 055:B5, Sigma-Aldrich) for 24 hours. The SNs were collected, centrifuged for 6 minutes at $500 \mathrm{~g}$, and added to AB1 cells in a 24-well plate $\left(2 \times 10^{4}\right.$ cells/well) for 48 hours. FeTTPS at $75 \mu \mathrm{M}$ (Calbiochem) was used as a catalyst for peroxynitrite decomposition. To quantify apoptosis, floating and adherent cells were pooled, washed twice with cold PBS, resuspended in $100 \mu \mathrm{L}$ of $1 \times$ annexin V buffer (Becton Dickinson), and incubated for 15 minutes with $5 \mu \mathrm{L}$ annexin V-FITC and propidium iodide $(1 \mu \mathrm{g} / \mathrm{mL}$, MilliporeSigma) reagent. Fluorescence emission (filter 530/30) was analyzed by flow cytometry using BD FACSAria, and data were analyzed with the FACSDiva software (Becton Dickinson).

Direct cytotoxicity assay. RAW264.7 macrophages (wild-type or knocked down for EZH2) were cultivated in a 24 -well plate $\left(5 \times 10^{4}\right.$ cells/well) in the presence or absence of apocynin, L-NMMA, or EPZ for 24 hours and then with LPS for 24 hours. AB1 cells (wild-type or knocked down for PGAM5) were labeled with $10 \mu \mathrm{M}$ CFSE (Abcam) according to the manufacturer's instructions, washed with complete RPMI 1640, and cocultivated for 48 hours at a 1:10 ratio with RAW264.7 macrophages. Cells in coculture were recorded by time-lapse microscopy using a Zeiss LSM 510 equipped with an environmental chamber maintained at $37^{\circ} \mathrm{C}$ in a humidified $5 \% \mathrm{CO}_{2}$ air atmosphere. Apoptosis of $\mathrm{CFSE}^{+} \mathrm{AB} 1$ cells was analyzed by using the annexin V-APC kit (Becton Dickinson). PD-1 expression on RAW264.7 macrophages was revealed with PE/Cy7-conjugated anti-mouse PD-1 antibody (clone RMP1-30, SONY Biotechnology). Fluorescence emission was analyzed by flow cytometry using an FACSAria (Becton Dickinson). Ten thousand $\mathrm{CFSE}^{+}$cells were collected and analyzed with the FACSDiva software.

For time-lapse microscopy, CFSE-labeled RAW264.7 and/or AB1 cells were cocultivated for 24 hours at a 1:1 ratio in the presence of annexin V-APC (Becton Dickinson), propidium iodide $(0.5 \mu \mathrm{M}$, MilliporeSigma), or PE/Cy7-conjugated anti-mouse PD-1 (RMP1-30, SONY Biotechnology). For blocking PD-1, RAW264.7 macrophages were incubated with anti-PD-1 antibody (10 $\mu \mathrm{g} / \mathrm{mL}$; clone 29F.1A12; InVivoMAb, BioXcell) or rat IgG2a isotype control (10 $\mu \mathrm{g} / \mathrm{mL}$, clone R35-95, BD) for 6 hours before cocultivation with AB1 cells. For blocking PD-L1, RAW264.7 cells were cocultivated with AB1 cells in the presence of anti-PD-L1 antibody (10 $\mu \mathrm{g} / \mathrm{mL}$; clone 10F.9G2; InVivoMAb, BioXcell,) or rat IgG2b isotype control $(10 \mu \mathrm{g} / \mathrm{mL}$, clone A95-1, BD). The cells were monitored by the IncuCyte S3 Live-Cell imaging system (Essen Bioscience) placed in an incubator maintained at $37^{\circ} \mathrm{C}$ in a humidified $5 \% \mathrm{CO}_{2}$ atmosphere. The number of annexin $\mathrm{V}^{+}$events on CFSE-labeled AB1 cells was determined every 10 minutes for 24 hours using the IncuCyte S3 Live-Cell imaging system.

Determination of ROS. Intracellular ROS production was measured using the cell-permeant $\mathrm{H}_{2} \mathrm{DCF}$ DA probe (Molecular Probes). RAW264.7 macrophages $\left(25 \times 10^{3}\right.$ cells/well of a 96-well microplate) were washed with PBS, incubated with $10 \mu \mathrm{M}$ of $\mathrm{H}_{2}$ DCFDA diluted in serum-free RPMI culture medium for 30 minutes at $37^{\circ} \mathrm{C}$, washed once with PBS, and further maintained in serum-free RPMI for 30 minutes. After lysis in PBS with $1 \%$ Triton X-100 $(v / v)$ for 10 minutes, $150 \mu \mathrm{L}$ of cell lysate was transferred into a black 96-well microplate (PerkinElmer). Fluorescence emission was determined by using a Victor ${ }^{3} \mathrm{~V} 1420$ multilabel counter (Perkin Elmer Wallac) with an excitation wavelength at $485 \mathrm{~nm}$ and an emission filter at $535 \mathrm{~nm}$.

Quantification of $N O$ production. The levels of nitrites $\left(\mathrm{NO}_{2}^{-}\right)$were measured using sulfanilamide and N-1-napthylethylenediamine dihydrochloride (Griess reaction assay, Promega). Cell culture SNs were incubated with Griess reagent for 15 minutes in the dark at room temperature. The optical density was measured at $546 \mathrm{~nm}$ with a Multiskan GO Microplate Spectrophotometer (Thermo Fisher Scientific). The concentrations of nitrites were derived by regression analysis using serial dilutions of sodium nitrite as a standard.

Analysis of tyrosine nitrosylation by confocal fluorescence microscopy. RAW264.7 cells were cultivated on coverslips $\left(5 \times 10^{4}\right.$ cells/well) in the presence or absence of LPS for 24 hours. Then, RAW264.7 cells were cocultivated with AB1 cells for 48 hours at a 10:1 ratio. Cells were fixed with 4\% paraformaldehyde in PBS 
for 15 minutes, permeabilized using 1\% Triton X-100 in PBS for 10 minutes, and blocked in PBS containing $10 \%$ FCS for 10 minutes. After a wash with PBS, cells were incubated for 1 hour at room temperature with rat anti-mouse F4/80 (Becton Dickinson, clone T45-2342) and rabbit anti-nitrotyrosine (A-21285, Thermo Fisher Scientific) antibodies. Then, cells were stained with Alexa Fluor 488 anti-rat IgG and Alexa Fluor 647 anti-rabbit IgG (Invitrogen) conjugates. Coverslips were washed and mounted in fluoroshield (MilliporeSigma). Images were acquired with a Zeiss LSM 510 confocal microscope equipped with $\times 40$ and $\times 63-1.4$ oil immersion objectives (Zeiss).

H3K27me3 immunofluorescence. RAW264.7 macrophages $\left(5 \times 10^{4}\right.$ cells/well of a 24 -well plate) were grown on coverslips and treated with EPZ for 48 hours. Then, cells were fixed with $4 \%$ paraformaldehyde for 15 minutes, permeabilized using 1\% Triton X-100 in PBS for 10 minutes, and blocked in PBS containing $10 \%$ FCS for 10 minutes. After a wash with PBS, cells were incubated for 1 hour at room temperature with a rabbit polyclonal antibody directed against H3K27me3 (07-449, MilliporeSigma) and an Alexa Fluor 488 anti-rabbit IgG conjugate (Invitrogen). Cells were stained with Draq5 (Invitrogen) and mounted with fluoroshield (MilliporeSigma). Images were acquired with a Zeiss LSM 510 confocal microscope equipped with $\times 40$ and $\times 63-1.4$ oil immersion objectives (Zeiss). For flow cytometry analysis, RAW264.7 macrophages were fixed with $4 \%$ paraformaldehyde for 15 minutes, permeabilized using $1 \%$ Triton X-100, and blocked in PBS containing 10\% FCS for 10 minutes. RAW264.7 macrophages were labeled with a rabbit polyclonal antibody directed against pan-H3 (RM188, MilliporeSigma) and mouse IgG3 anti-H3K27me3 (6002, Abcam) for 1 hour. The cells were washed and incubated with Alexa Fluor 647 anti-rabbit IgG and Alexa Fluor 488 anti-mouse IgG (Invitrogen) conjugates. Isotype-matched antibodies were used as controls. Fluorescence emission was analyzed by flow cytometry using a BD FACSAria, and data were analyzed with the FACSDiva software.

RNA analysis. Total RNA was prepared from whole cells using NucleoSpin RNA Plus Kit (Macherey-Nagel) according to the manufacturer's instructions. After reverse transcription using FastGene Scriptase II cDNA Kit (Nippon Genetics), the abundance of transcripts was assessed by real-time qPCR analysis using the Takyon SYBR MasterMix (Eurogentec) and gene-specific primer sets: PGAM5 (5'-ATCTGGAGAAGACGAGTTGACA-3'; 5'-CCTGTTCCCGACCTAATGGT-3') and GAPDH (5'-CGGAGTCAACGGATTTGGTCGTAT-3'; 5'-AGCCTTCTCCATGGTGGTGAAGAC-3'). Samples were amplified in triplicate on each plate, and data were analyzed using LightCycler 480 Software (Roche Diagnostics). The fluorescence intensities were quantified with LightCycler 480 Software SW 1.5.1. Normalization was performed using mouse GAPDH as an internal control, and relative gene expression was calculated using the comparative $2^{-\Delta \Delta \mathrm{Ct}}$ method.

ChIP. ChIP of the PD-1 promoter using anti-H3K27me3 antibody (07-449, MilliporeSigma) was performed using the iDeal ChIP for histones (Diagenode). Briefly, RAW264.7 cells were cross-linked with 1\% paraformaldehyde for 10 minutes at room temperature. Chromatin was fragmented by sonication using a Bioruptor (Diagenode) to an average length of 200-500 base pairs. Antibody-conjugated beads were then added to chromatin samples and incubated overnight at $4^{\circ} \mathrm{C}$. Chromatin was eluted from the beads, and DNA was purified according to the manufacturer's instructions. Precipitated DNA was amplified with Takyon qPCR SYBR MasterMix (Eurogentec) on a LightCycler 480 thermocycler (Roche Diagnostics) and primers specific for regions B (5'-CCACCTCTAGTTGCCTGTTCTC-3' and 5'-CCTCACCTCCTGCTTGTCTCTC-3') and C (5'-GTGAGACCCACACATCTCATTGC-3' and 5'-ATTCCCATCCATACCTTGCTCC-3') of the PD-1 promoter (GeneID 18566).

Western blot. Whole-cell lysates were prepared using RIPA buffer $(50 \mathrm{mM}$ Tris- $\mathrm{HCl}$ at $\mathrm{pH} 8,150 \mathrm{mM}$ $\mathrm{NaCl}, 1 \% \mathrm{NP}-40,0.1 \%$ SDS, $0.5 \%$ sodium deoxycholate, and protease inhibitors). After protein quantification (Pierce BCA Protein Assay), $50 \mu \mathrm{g}$ of lysate was separated by electrophoresis on SDS-PAGE gels and blotted onto nitrocellulose. Membranes were incubated with antibodies against PD-1 (84651, Cell Signaling Technology), $\alpha$-tubulin (clone B5-1-2, MilliporeSigma), or EZH2 (clone D2C9, Cell Signaling Technology), then with the appropriate secondary antibodies conjugated with horseradish peroxidase (HRP) (antirabbit 7074, Cell Signaling Technology; anti-mouse P0260, Dako). After incubation with enhanced luminescence (Pierce), protein bands were quantified using ImageJ software (NIH).

Animal experiments. All procedures were approved by the Ethical Review Board (protocol 1564) and performed according to the Federation of Laboratory Animal Science Association (FELASA) guidelines. Male wild-type BALB/c mice (5-7 weeks old) from the GIGA mouse facility platform (University of Liège) were inoculated with syngeneic RAW264.7 macrophages and AB1/AB12 mesothelioma cells. RAW264.7 macrophages were cultured with the EZH2 inhibitor (10 $\mu \mathrm{M} \mathrm{EPZ)} \mathrm{for} 24$ hours, then activated with LPS (1 
$\mu \mathrm{g} / \mathrm{mL}$ ) for an additional day as indicated in Figure 4A. For blocking PD-1, RAW264.7 macrophages were incubated with anti-PD-1 antibody $(10 \mu \mathrm{g} / \mathrm{mL}$; InVivoMAb, BioXcell) or rat IgG2a isotype control (10 $\mu \mathrm{g} /$ $\mathrm{mL}$, Becton Dickinson) for 6 hours before inoculation. After 3 washes with PBS, macrophages were coimplanted SC with $2 \times 10^{6} \mathrm{AB} 1$ or AB12 cells into the flanks of BALB/c mice at a 1:3 ratio. Tumor volumes were calculated weekly using the following formula: $4 / 3 \times \pi \times(\text { diameter } / 2)^{3}$. Groups of at least 6 mice were tested in each experimental condition.

Immunohistochemistry of tumors. Tumors from each flank were collected, formalin fixed, and paraffin embedded. Sections were cut at $4 \mu \mathrm{m}$ of thickness and mounted on microscope slides, dewaxed in xylene (MilliporeSigma), and rehydrated in graded ethanol bath (MilliporeSigma). Sections were stained with hematoxylin and eosin (MilliporeSigma). For immunofluorescence, slides were preincubated with PBS containing $0.5 \%$ bovine serum albumin (BSA; MilliporeSigma) for 1 hour to reduce nonspecific background. After rinsing with PBS with $0.5 \%$ BSA, samples were incubated with rat anti-mouse F4/80 (Becton Dickinson) and rabbit anti-active caspase-3 (Becton Dickinson) primary antibodies for 1 hour at room temperature. After washing with PBS, slices were incubated with donkey anti-rat Alexa Fluor 488, chicken anti-rabbit Alexa Fluor 546, and DAPI for 30 minutes. Slides were imaged with an Olympus FSX100 epifluorescence microscope by performing $3 \times 3$ segmentation at an original magnification of $\times 40$. The cleaved caspase- 3 fluorescence was quantified from 25 images by using ImageJ and FSX100 software. For immunohistochemistry, slides were stained with anti-CD3 (RM9107-S, Thermo Fisher Scientific) or antiPD-1 (84651, Cell Signaling Technology) monoclonal antibodies. After incubation with an HRP conjugate, sections were stained with hematoxylin and analyzed by using ImageJ and QuPath software (45).

Statistics. The normal distribution, equal variance, and comparison of means were calculated using GraphPad Prism 5 and Minitab 16 (University of Liege) software. Means within a data set were compared by 1-way ANOVA followed by Tukey's multiple-comparisons test. Parametric paired 2-tailed Student's $t$ test was used to determine the difference between 2 paired distributions. Statistical significance between 2 non-Gaussian distributions was calculated by using the 2-tailed Mann-Whitney $U$ test. The analysis of tumor growth was performed using 2-way ANOVA and Bonferroni's post tests. Survival curves were compared by using log-rank test $\left(\chi^{2}\right)$. For immunohistochemistry, statistical analysis was performed using nonparametric Friedman's test followed by Dunn's multiple-comparisons test. Data were considered statistically significant $\left({ }^{*}\right)$, very statistically significant $\left({ }^{* *}\right)$, and highly statistically significant $\left({ }^{* *}\right)$ at $P<0.05, P<$ 0.01 , and $P<0.001$, respectively.

Study approval. All procedures were approved by the ethical review board of the University Hospital of Liege (protocol 1564) and performed according to the FELASA guidelines.

\section{Author contributions}

$\mathrm{MH}$ and LW designed experiments, analyzed data, and drafted the manuscript. $\mathrm{HG}, \mathrm{GBH}, \mathrm{CH}, \mathrm{BD}$, and RL contributed to experiments.

\section{Acknowledgments}

This work was supported by the Belgian Foundation against Cancer, the Fonds National de la Recherche Scientifique (FNRS), the Télévie, and the Fondation Léon Fredericq. We thank the GIGA technological platforms, in particular the immunohistology, imaging, and animal facilities. We are grateful to Christelle Gillissen, Nathalie Renotte, and Jean-Rock Jacques for technical expertise and Pascale Hubert for helpful discussions. $\mathrm{MH}$ and HG are supported by fellowships of the Belgian Foundation against Cancer and the Télévie. CH (research fellow) and LW (research director) are members of the FNRS.

Address correspondence to: Luc Willems, Molecular and Cellular Epigenetics, Interdisciplinary Cluster for Applied Genoproteomics (GIGA), University of Liège, B34, 1 avenue de l'Hôpital, 4000 Sart-Tilman Liège, Belgium. Phone: 32.81.622152; Email: 1uc.willems@uliege.be.

1. Yap TA, Aerts JG, Popat S, Fennell DA. Novel insights into mesothelioma biology and implications for therapy. Nat Rev Cancer. 2017;17(8):475-488.

2. Bianchi C, Bianchi T. Global mesothelioma epidemic: trend and features. Indian J Occup Environ Med. 2014;18(2):82-88.

3. Price B, Ware A. Time trend of mesothelioma incidence in the United States and projection of future cases: an update based on SEER data for 1973 through 2005. Crit Rev Toxicol. 2009;39(7):576-588. 
4. Vogelzang NJ, et al. Phase III study of pemetrexed in combination with cisplatin versus cisplatin alone in patients with malignant pleural mesothelioma. J Clin Oncol. 2003;21(14):2636-2644.

5. Bueno R, et al. Comprehensive genomic analysis of malignant pleural mesothelioma identifies recurrent mutations, gene fusions and splicing alterations. Nat Genet. 2016;48(4):407-416.

6. LaFave LM, et al. Loss of BAP1 function leads to EZH2-dependent transformation. Nat Med. 2015;21(11):1344-1349.

7. Italiano A, et al. Tazemetostat, an EZH2 inhibitor, in relapsed or refractory B-cell non-Hodgkin lymphoma and advanced solid tumours: a first-in-human, open-label, phase 1 study. Lancet Oncol. 2018;19(5):649-659.

8. Cornelissen $\mathrm{R}$, et al. Intratumoral macrophage phenotype and $\mathrm{CD} 8^{+} \mathrm{T}$ lymphocytes as potential tools to predict local tumor outgrowth at the intervention site in malignant pleural mesothelioma. Lung Cancer. 2015;88(3):332-337.

9. Chéné AL, et al. Pleural effusions from patients with mesothelioma induce recruitment of monocytes and their differentiation into M2 macrophages. J Thorac Oncol. 2016;11(10):1765-1773.

10. Gordon S, Plüddemann A, Martinez Estrada F. Macrophage heterogeneity in tissues: phenotypic diversity and functions. Immunol Rev. 2014;262(1):36-55.

11. Mantovani A, Allavena P. The interaction of anticancer therapies with tumor-associated macrophages. J Exp Med. 2015;212(4):435-445.

12. Beatty GL, et al. CD40 agonists alter tumor stroma and show efficacy against pancreatic carcinoma in mice and humans. Science. 2011;331(6024):1612-1616.

13. O'Sullivan T, et al. Cancer immunoediting by the innate immune system in the absence of adaptive immunity. JExp Med. 2012;209(10):1869-1882.

14. Schreiber RD, Old LJ, Smyth MJ. Cancer immunoediting: integrating immunity's roles in cancer suppression and promotion. Science. 2011;331(6024):1565-1570.

15. Mantovani A, Sica A, Sozzani S, Allavena P, Vecchi A, Locati M. The chemokine system in diverse forms of macrophage activation and polarization. Trends Immunol. 2004;25(12):677-686.

16. Ivashkiv LB. Epigenetic regulation of macrophage polarization and function. Trends Immunol. 2013;34(5):216-223.

17. Mezzapelle R, et al. Human malignant mesothelioma is recapitulated in immunocompetent BALB/c mice injected with murine AB cells. Sci Rep. 2016;6:22850.

18. Gordon SR, et al. PD-1 expression by tumour-associated macrophages inhibits phagocytosis and tumour immunity. Nature. 2017;545(7655):495-499.

19. Hegmans JP, Hemmes A, Hammad H, Boon L, Hoogsteden HC, Lambrecht BN. Mesothelioma environment comprises cytokines and T-regulatory cells that suppress immune responses. Eur Respir J. 2006;27(6):1086-1095.

20. Izzi V, et al. Differential effects of malignant mesothelioma cells on THP-1 monocytes and macrophages. Int J Oncol. 2009;34(2):543-550

21. Matzinger P. Tolerance, danger, and the extended family. Annu Rev Immunol. 1994;12:991-1045.

22. Matzinger P. Friendly and dangerous signals: is the tissue in control? Nat Immunol. 2007;8(1):11-13.

23. Kaarteenaho-Wiik R, Soini Y, Pöllänen R, Pääkkö P, Kinnula VL. Over-expression of tenascin-C in malignant pleural mesothelioma. Histopathology. 2003;42(3):280-291.

24. Hegmans JP, et al. Proteomic analysis of exosomes secreted by human mesothelioma cells. Am J Pathol. 2004;164(5):1807-1815

25. Lotze MT, Tracey KJ. High-mobility group box 1 protein (HMGB1): nuclear weapon in the immune arsenal. Nat Rev Immunol. 2005;5(4):331-342.

26. Yang $\mathrm{H}$, et al. A critical cysteine is required for HMGB1 binding to Toll-like receptor 4 and activation of macrophage cytokine release. Proc Natl Acad Sci U S A. 2010;107(26):11942-11947.

27. Yang H, et al. Programmed necrosis induced by asbestos in human mesothelial cells causes high-mobility group box 1 protein release and resultant inflammation. Proc Natl Acad Sci U S A. 2010;107(28):12611-12616.

28. Jube S, et al. Cancer cell secretion of the DAMP protein HMGB1 supports progression in malignant mesothelioma. Cancer Res. 2012;72(13):3290-3301.

29. Feng M, et al. Macrophages eat cancer cells using their own calreticulin as a guide: roles of TLR and Btk. Proc Natl Acad Sci U S A. 2015;112(7):2145-2150.

30. Williams CB, Yeh ES, Soloff AC. Tumor-associated macrophages: unwitting accomplices in breast cancer malignancy. NPJ Breast Cancer. 2016;2:15025.

31. Engels F, Renirie BF, Hart BA, Labadie RP, Nijkamp FP. Effects of apocynin, a drug isolated from the roots of Picrorhiza kurroa, on arachidonic acid metabolism. FEBS Lett. 1992;305(3):254-256.

32. Kleinert H, Pautz A, Linker K, Schwarz PM. Regulation of the expression of inducible nitric oxide synthase. Eur J Pharmacol. 2004;500(1-3):255-266.

33. Denicola A, Souza JM, Radi R. Diffusion of peroxynitrite across erythrocyte membranes. Proc Natl Acad Sci U S A. 1998;95(7):3566-3571.

34. Alvarez MN, Trujillo M, Radi R. Peroxynitrite formation from biochemical and cellular fluxes of nitric oxide and superoxide. Meth Enzymol. 2002;359:353-366.

35. Fraszczak J, et al. Peroxynitrite-dependent killing of cancer cells and presentation of released tumor antigens by activated dendritic cells. J Immunol. 2010;184(4):1876-1884.

36. Lakomy D, et al. Cytotoxic dendritic cells generated from cancer patients. J Immunol. 2011;187(5):2775-2782.

37. Holze C, et al. Oxeiptosis, a ROS-induced caspase-independent apoptosis-like cell-death pathway. Nat Immunol. 2018;19(2):130-140.

38. Li S, Xu X, Jiang M, Bi Y, Xu J, Han M. Lipopolysaccharide induces inflammation and facilitates lung metastasis in a breast cancer model via the prostaglandin E2-EP2 pathway. Mol Med Rep. 2015;11(6):4454-4462.

39. Abdi K, Singh NJ, Matzinger P. Lipopolysaccharide-activated dendritic cells: "exhausted" or alert and waiting? J Immunol. 2012;188(12):5981-5989.

40. Arya SB, Kumar G, Kaur H, Kaur A, Tuli A. ARL11 regulates lipopolysaccharide-stimulated macrophage activation by promoting mitogen-activated protein kinase (MAPK) signaling. J Biol Chem. 2018;293(25):9892-9909.

41. Rajaiah R, Perkins DJ, Polumuri SK, Zhao A, Keegan AD, Vogel SN. Dissociation of endotoxin tolerance and differentiation 
of alternatively activated macrophages. J Immunol. 2013;190(9):4763-4772.

42. López-Collazo E, del Fresno C. Pathophysiology of endotoxin tolerance: mechanisms and clinical consequences. Crit Care. 2013;17(6):242.

43. Kemp CD, et al. Polycomb repressor complex-2 is a novel target for mesothelioma therapy. Clin Cancer Res. 2012;18(1):77-90.

44. Zingg D, et al. The histone methyltransferase Ezh2 controls mechanisms of adaptive resistance to tumor immunotherapy. Cell Rep. 2017;20(4):854-867.

45. Bankhead P, et al. QuPath: Open source software for digital pathology image analysis. Sci Rep. 2017;7(1):16878. 